\title{
SEMIPARAMETRIC IDENTIFICATION OF STRUCTURAL DYNAMIC OPTIMAL STOPPING TIME MODELS
}

Le-Yu Chen

THE INSTITUTE FOR FISCAL STUDIES DEPARTMENT OF ECONOMICS, UCL cemmap working paper CWP06/07 


\title{
Semiparametric Identification of Structural Dynamic Optimal Stopping Time Models ${ }^{1}$
}

\author{
Le-Yu Chen \\ Department of Economics \\ University College London \\ E-mail : le-yu.chen@ucl.ac.uk
}

January 2007

\section{[Abstract]}

This paper presents new identification results for the class of structural dynamic optimal stopping time models that are built upon the framework of the structural discrete Markov decision processes proposed by Rust (1994). We demonstrate how to semiparametrically identify the deep structural parameters of interest in the case where the utility function of an absorbing choice in the model is parametric but the distribution of unobserved heterogeneity is nonparametric. Our identification strategy depends on availability of a continuous observed state variable that satisfies certain exclusion restrictions. If such excluded variable is accessible, we show that the dynamic optimal stopping model is semiparametrically identified using control function approaches.

KEYWORDS : structural dynamic discrete choice models, semiparametric identification, optimal stopping time models

\section{Introduction}

Over the decades, structural estimation of dynamic discrete choice models has been more and more employed in empirical economics and for the assessment of various policies of interest. Well-known empirical applications include Wolpin (1984) and Holz and Miller (1993) in fertility; Pakes (1986) in patent renewal; Rust (1987), Das (1992), Cooper, Haltiwanger, and Power (1999), and Adda and Cooper (2000) in capital retirement and replacement; Wolpin (1987) in job search; Berkovec and Stern (1991), Daula and Moffitt (1995), Rust and Phelan (1997), and Karlstrom, Palm and Svenssonin (2004) in retirement from labor force; Erdem and Keane (1996) in brand choice; Keane and Wolpin (1997) in career choice; Eckstein and Wolpin (1999) in education choice. See Eckstein and Wolpin (1989) for a survey of the earlier empirical work on dynamic discrete choice models and Wolpin (1996) for the use of such models in public policy evaluation.

\footnotetext{
${ }^{1}$ I am grateful to my advisor, Dr. Simon Lee for many helpful comments on this paper and valuable suggestions at various stages of my research.
} 
Structural estimation of dynamic discrete choice models is attractive to researchers because it tightly links economic theories of rational decision making under uncertainty in a dynamic setting to the interpretation and prediction of the stochastic process that generates the economic data of interest. The parameters in the model and the estimation methods are structural in the sense that they are derived from solutions of an explicitly postulated economic behavioral model. Hence structural estimation avoids Lucas critique about the use of reduced form analysis and it can allow researchers to simulate the consequences of vaious policy experiments after the underlying structural parameters are estimated.

However, a common feature in the structural estimation literature is the parametric specification of the underlying structural objects such as utility functions, transition probabilities of state variables and distributions of the unobservables. Estimated results from parametric models may be sensitive to changes of specification and hence suffer from problems of misspecification. Therefore, later work in the literature tries to study the nonparametric identification of the dynamic discrete choice model. An influential work by Rust (1994) shows that the dynamic discrete choice model is nonparametrically unidentified. Magnac and Thesmar (2002) extend Rust's framework and show that if the history of observed variables is discrete, the dynamic discrete choice model is nonidentified. They then further characterize the degree of nonidentification and show that the model is identified subject to ad hoc assumptions on distributions of unobservables and functional forms of agents' preferences. In particular, their identification result indicates that parametric specifications on the distributions of unobservables are indispensable for identifying the deep structural parameters of interest, which consequently motivates the maximum likelihood estimation approach to most of the empirical work on structural estimation of the dynamic discrete choice model.

This paper builds on the framework of Rust (1994) and Magnac and Thesmar (2002) and develops new identification results for the case where the distribution of unobservables is nonparametric. We mainly focus on the identification analysis of the optimal stopping time model which is a subclass of dynamic discrete choice models. The reason is twofolds. First, the optimal stopping time model has appeared as an important modeling framework in many empirical applications in which the agent faces an irreversible choice. See for example Pakes (1986) in patent renewal, Das (1992) in capital utilization and retirement, Daula and Moffitt (1995) in military reenlistment, and Rothwell and Rust (1997) in nuclear plant operation models. The distribution of unobservables in these models is often parametrically specified and estimated. Therefore, it is important to investigate the degree of identification for such models when the researcher does not hold a priori information on the distribution of unobservables. Second, the dynamic discrete choice model is generally highly nonlinear and the solution of the model is often implicitly defined as the fixed point of a system of Bellman equations that characterize the agent's 
rational behavior. Therefore, identification analysis of dynamic discrete choice models is quite a nontrivial task when the distribution of unobservables is nonparametric. Instead of trying to completely resolve the identification of the general model, we study the optimal stopping time model in which we can exploit the identification power arising from the irreversible (absorbing) choice restriction. We present the identification analysis of the optimal stopping time model while leaving that of the general case for further research to which the analysis in this paper could be useful.

As in static discrete choice models, the dynamic discrete choice model is not nonparametrically identified when all regressors (observed state variables) are discrete and the distribution of unobservables is unknown and continuous. To allow for nonparametric distribution of the unobservables, we assume the availability of continuous observables so that we can still gain identification by exploiting continuous variation from observed continuous state variables. However, as noted in Magnac and Thesmar (2002), the dynamic discrete choice model is still unidentified even if the distribution of unobservables is given a priori. To secure identification, the researcher needs to further assume the functional form of the utility for one of the choices. When the distribution of unobservables is nonparametric, as will be evident later the assumption on the utility function seems inevitable to secure identification. Therefore, through the paper the identification analysis is semiparametric in the sense that one of the utility functions of the choices is given a priori. Under this assumption, we show that the optimal stopping time model is semiparametrically identified if there is an exclusion restriction that provides identification power when information about distribution of unobservables is not available. When there is no exclusion restriction, we show that the optimal stopping time model is not identified even when the utility function of one choice is known. Therefore, in this respect, our analysis provides semiparametric identification for the dynamic counterpart to the already well developed semiparametric static discrete choice models in which preference shock distributions are nonparametric but systematic utility functions are restricted to certain function space ${ }^{2}$.

In the identification analysis of structural dynamic optimal stopping time models, our work is related to the analysis in Heckman and Navarro (2006). In their paper, they consider semiparametric identification of a variety of reduced form optimal stopping time models and a finite horizon structural optimal stopping model. They identify the structural model by solving it backwards. Under the finite horizon restriction and their limit set identification strategies which generalize Taber's (2000) identification-at-infinity arguments, they can achieve

\footnotetext{
${ }^{2}$ See Manski (1975, 1985), Cosslett (1983), Stoker (1986), Klein and Spady (1988), and Ichimura (1993) for studies of semiparametric identification and estimation of static discrete choice models in which the random shock distribution is nonparametric but systematic utility is parametric. Matzkin (1992, 1993) and Lewbel and Linton (2005) consider nonparametric discrete choice models by further relaxing parametric assumptions on the systematic utility to a function space defined under certain shape restrictions such as linear homogeneity that are usually motivated from economic theories.
} 
semiparametric identification of the model while allowing for richer time series dependence between nonparametric unobservables than is assumed in Rust's (1994) framework. However, such backward solving procedure cannot apply in infinite horizon models and the limiting set required for their identification may also be demanding in practical applications ${ }^{3}$. In this paper, we follow Rust's (1994) conditional independence assumption that precludes time series dependence between unobservables to achieve identification in the infinite horizon model. Our identification strategy is based on solving the fixed point of the Bellman equations in the model and hence is applicable to both finite and infinite horizon models. Since the conditional independence framework in Rust (1994) has been popular in empirical work, it is important to understand the identification problem of infinite horizon models under this assumption before considering more general serial dependence between unobservables. Furthermore, such setup also includes an important class of models in which rational expectation assumption on agents' belief can be justified from a learning process based on observations (Magnac and Thesmar 2002).

The rest of the paper is organized as follows. Section 2 presents the framework and assumptions of the optimal stopping time model. Section 3 study the semiparametric identification of the model. Section 4 concludes the paper.

\section{The framework and assumptions}

Time is discrete and indexed by $t \in\{1,2, \ldots\}$. Consider an economic agent with intertemporal utility that is additive separable over time. At each period, the agent makes a decision over two mutually exclusive choices ${ }^{4}$, denoted as choice 0 and choice 1 . One of these two choices is assumed absorbing or irreversible in the sense that once it is chosen, the agent will never deviate from this choice in the rest of the decision horizon. Let choice 0 be the absorbing choice. Denote as $s_{t}$ the state variables at time $t$ that the agent considers in this optimal stopping time model. From the econometrician's point of view, some components of $s_{t}$ are observables that are denoted by $x_{t}$. Others are unobserved random shocks $\varepsilon_{t} \equiv\left(\varepsilon_{0, t}, \varepsilon_{1, t}\right)$ for each choice. Therefore, $s_{t}=\left(x_{t}, \varepsilon_{t}\right)$. At the beginning of each period $t, s_{t}$ is revealed to the agent who then chooses $d_{t} \in\{0,1\}$ and receives the instantaneous return $u_{t}\left(d_{t}, s_{t}\right)$. However, next period state variables $s_{t+1}$ are still uncertain to the agent. Following Rust (1994)'s dynamic Markov discrete decision process framework, we assume the transition of state variables follows controlled

\footnotetext{
${ }^{3}$ In the empirical example of their paper (Heckman and Navarro 2006), they report that they do not have the required limit sets in their data.

${ }^{4}$ To present the main idea, we focus on binary choice models. It is possible to generalize the result of this paper to the multinomial choice context. We leave such generalization for further research work.
} 
first-order Markov property. In other words, the next period state variables $s_{t+1}$ are drawn based on the Markov transition probability density $f_{s}\left(s_{t+1} \mid s_{t}, d_{t}\right)$. The agent has belief, $\mu\left(s_{t+1} \mid s_{t}, d_{t}\right)$ about the evolution of the state variables. We assume that the agent's belief is rational in the sense that it coincides with the true transition probability $f_{s}\left(s_{t+1} \mid s_{t}, d_{t}\right)^{5}$. To proceed, as in Rust (1994) and Magnac and Thesmar (2002, pp. 802-804), we make the following assumptions on the agent's preference and laws of motion of the states.

\section{Assumption M1 (Additive Separability) :}

for $k \in\{0,1\}$ and for all $t, u_{t}\left(k, s_{t}\right) \equiv u_{k, t}\left(s_{t}\right)=u_{k, t}^{*}\left(x_{t}\right)+\varepsilon_{k, t}$.

Assumption M2 (Conditional Independence) :

for all $t, f_{s}\left(x_{t+1}, \varepsilon_{t+1} \mid x_{t}, \varepsilon_{t}, d_{t}\right)=f_{\varepsilon}\left(\varepsilon_{t+1}\right) f_{x}\left(x_{t+1} \mid x_{t}, d_{t}\right)$.

The additive separability assumption allows us to decompose the utility into a systematic part that depends only on observable state variables and a preference shock that is unobserved to the econometrician. This is a standard assumption in static discrete choice models ${ }^{6}$. M2 assumes that the unobservables $\varepsilon_{t}$ are i.i.d. exogenous random shocks to the model and future values of the observed states $x_{t+1}$ can depend on the current control variable $d_{t}$ and values of current observed states $x_{t}$ but they do not direct depend on current values of the exogenous shocks $\varepsilon_{t}$. Since M2 restricts the time series dependence of unobserved state variables, it precludes unobserved persistent heterogeneity and dynamic selection bias discussed in Cameron and Heckman (1998), Taber (2000), and Heckman and Navarro $(2006)^{7}$. However, general time series dependence between observables are allowed. Besides, preference shocks can also be dependent across alternatives. Note that M2 allows rational expectation belief to be derived from a learning process based on observations in the sense that the agent can infer joint distribution of next period states based on observable information from other agents and on the assumption that he knows distribution of his private signal $\varepsilon_{t}$ (Manski 1993, Magnac and Thesmar 2002).

Let $\beta \in[0,1)$ be the discount factor. Assuming at each period choices are made to maximize the agent's expected life time utility, under M1, M2 and rational expectation belief, by Bellman principle of optimality, $\pi_{t}$, the policy function (the optimal decision rule) which depends on previous period control variable $d_{t-1}$ due to the

\footnotetext{
${ }^{5}$ In general, the agent's belief $\mu\left(s_{t+1} \mid s_{t}, d_{t}\right)$ is subject to his degree of rationality and hence may not necessarily represent the true law of motion of the states. However, identification of a general belief requires more structural assumptions about the agent's belief formation process.

${ }^{6}$ See Vytlacil $(2002,2005)$ for discussions about the use of additively separable utility functions in static discrete choice models.

${ }^{7}$ See Rust (1994) for a detailed and graphical explanation of controlled Markov process under such conditional independence assumption.
} 
optimal stopping feature of the model, can be characterized as the follows.

$$
\pi_{t}\left(s_{t}, d_{t-1}\right)=\underset{k \in\{0,1\}}{\arg \max }\left\{v_{k, t}\left(s_{t}\right)\right\} \text { subject to } k \leq d_{t-1} \cdot{ }^{8}
$$

and the value function at each period $t$ can be obtained via the following recursive expressions.

$$
v_{t}\left(s_{t}, d_{t-1}\right)=\pi_{t}\left(s_{t}, d_{t-1}\right) v_{1, t}\left(s_{t}\right)+\left(1-\pi_{t}\left(s_{t}, d_{t-1}\right)\right) v_{0, t}\left(s_{t}\right),
$$

where

$$
v_{k, t}\left(s_{t}\right) \equiv u_{k, t}\left(s_{t}\right)+\beta E\left(v_{t+1}\left(s_{t+1}, k\right) \mid s_{t}, d_{t}=k\right) \text { for } k \in\{0,1\} .
$$

Assuming the agent starts from non-absorbing choice such that $\delta_{0}=1$, then under rationality the time series of observed choice is the sequence of optimal decisions $\left\{\delta_{t}=\pi_{t}\left(s_{t}, \delta_{t-1}\right)\right\}$ that follows the law of motion as follows.

$$
\delta_{t}=1\left\{v_{1, t}\left(s_{t}\right) \geq v_{0, t}\left(s_{t}\right)\right\} \delta_{t-1} .
$$

It is clear that under M1 and M2, for $k \in\{0,1\}$ and for all $t$, there is $v_{k, t}^{*}\left(x_{t}\right)$, which is a function of $x_{t}$ only, such that the choice specific value functions, $v_{k, t}\left(s_{t}\right)$ can be written as :

for $k \in\{0,1\}$ and for all $t$,

$$
v_{k, t}\left(s_{t}\right)=v_{k, t}^{*}\left(x_{t}\right)+\varepsilon_{k, t},
$$

where

$$
v_{k, t}^{*}\left(x_{t}\right)=u_{k, t}^{*}\left(x_{t}\right)+\beta E\left(v_{t+1}\left(s_{t+1}, k\right) \mid x_{t}, d_{t}=k\right) .
$$

Note that M1 together with M2 gives additive separability to the choice specific value functions, which greatly facilitates the development of identification and estimation methods in dynamic discrete choice models ${ }^{9}$.

For the rest of the paper, we will suppress the time index from the functions and variables. This can be justified since we can put the time index as part of the observed state variables. Furthermore, if we set the instantaneous utility to be zero for any $t>T$, then we can also represent a finite horizon model as an infinte horizon model. Denote variables $y$ and $y^{\prime}$ indicate the current and next period objects, respectively. Under this notation, we can

\footnotetext{
${ }^{8}$ When distribution of $\varepsilon_{t}$ is continous (see assumption M4), ties between the two choice specific value functions do not occur almost surely so that there is a unique solution to this optimization problem.

${ }^{9}$ Identification results for dynamic models with persistent unobserved state variables are generally negative (Magnac and Thesmar 2002). Besides, since violation of conditional independence implies that the entire history of unobserved state variables may enter value functions, due to the need of integrating these unobservables out, even in completely parametric setup, estimation of such models is in general so complicated that they are rarely used in practice (Rust 1994).
} 
formulate the model as follows. For $k \in\{0,1\}$,

$$
\begin{aligned}
v\left(s^{\prime}, d\right) & =\pi^{\prime}\left(s^{\prime}, d\right) v_{1}\left(s^{\prime}\right)+\left(1-\pi^{\prime}\left(s^{\prime}, d\right)\right) v_{0}\left(s^{\prime}\right) \\
u_{k}(s) & =u_{k}^{*}(x)+\varepsilon_{k} \\
v_{k}(s) & =v_{k}^{*}(x)+\varepsilon_{k} \\
v_{k}^{*}(x) & =u_{k}^{*}(x)+\beta E\left(v\left(s^{\prime}, k\right) \mid x, d=k\right) \\
\pi\left(s^{\prime}, d\right) & =1\left\{v_{1}\left(s^{\prime}\right) \geq v_{0}\left(s^{\prime}\right)\right\} d
\end{aligned}
$$

Let $\Delta v^{*}(x) \equiv v_{1}^{*}(x)-v_{0}^{*}(x)$ and $\Delta \varepsilon \equiv \varepsilon_{1}-\varepsilon_{0}$. Using the following lemma, we can further rewrite (10) to get a more convenient representation of the choice specific value functions.

Lemma 1 Under M1 and M2, the systematic Bellman equations (10) can be written as the follows.

$$
v_{1}^{*}(x)=u_{1}^{*}(x)+\beta E\left(v_{0}^{*}\left(x^{\prime}\right)+\varepsilon_{0}^{\prime} \mid x, d=1\right)+\beta\left[E\left(\Delta v^{*}\left(x^{\prime}\right) \pi\left(s^{\prime}, 1\right) \mid x, d=1\right)+E\left(\Delta \varepsilon^{\prime} \pi\left(s^{\prime}, 1\right) \mid x, d=1\right)\right]
$$

and

$$
v_{0}^{*}(x)=u_{0}^{*}(x)+\beta E\left(v_{0}^{*}\left(x^{\prime}\right)+\varepsilon_{0}^{\prime} \mid x, d=0\right)
$$

Proof. Lemma 1 follows immediately by putting equations (7) and (11) into (10).

Equation (12) expresses the value function for choice 1 as the sum of three terms. The first term on the right hand side of (12) is the instantaneous utility the agent receives when he makes choice 1 . The second term is the discounted expected future value when at next period the agent makes choice 0 given that his current action is choice 1 . The third term is the discounted expected gain when it is optimal for the agent to deviate from choice 0 at next period given that his current action is choice 1. Equation (13) interprets the value function for choice 0 in a similar fashion except that the term of the expected gain vanishes due to the behavioral restriction that choice 0 is absorbing and hence deviation is not allowed once choice 0 is made at previous period. The reduced form equation of this model is the conditional choice probability, $P\left(\delta^{\prime}=1 \mid x^{\prime}, \delta=1\right)=P\left(\Delta v^{*}\left(x^{\prime}\right)+\Delta \varepsilon^{\prime} \geq 0 \mid x^{\prime}, \delta=1\right)$. It is clear that the distribution of $\Delta \varepsilon$ is more critical than the joint distribution of $\varepsilon=\left(\varepsilon_{i}\right)_{i \in\{0,1\}}$ in characterizing the reduced form equation. Furthermore, if $E\left(\varepsilon_{0}\right)$ is normalized to be zero, then the systematic Bellman equations (12) and (13), as we will show later, will be completely characterized by the distribution of $\Delta \varepsilon$.

Assumption $M 3: E\left(\varepsilon_{0}\right)=0$. 
M3 provides location normalization for the random shock of the absorbing choice ${ }^{10}$. By M2, we have $E\left(\varepsilon_{0}^{\prime} \mid x, d=\right.$ $0)=E\left(\varepsilon_{0}^{\prime}\right)$ so that under M3 we can further simplify the systematic Bellman equations (12) and (13) as follows.

$$
v_{1}^{*}(x)=u_{1}^{*}(x)+\beta E\left(v_{0}^{*}\left(x^{\prime}\right) \mid x, d=1\right)+\beta\left[E\left(\Delta v^{*}\left(x^{\prime}\right) \pi\left(s^{\prime}, 1\right) \mid x, d=1\right)+E\left(\Delta \varepsilon^{\prime} \pi\left(s^{\prime}, 1\right) \mid x, d=1\right)\right]
$$

and

$$
v_{0}^{*}(x)=u_{0}^{*}(x)+\beta E\left(v_{0}^{*}\left(x^{\prime}\right) \mid x, d=0\right)
$$

To proceed, we need further assumptions on the distribution of $\Delta \varepsilon$.

Assumption $M_{4}:-\Delta \varepsilon$ has finite first moment and strictly increasing and absolutely continuous (with respect to Lebesgue measure) distribution function $G$ that induces a density function $g$, whose support is $[\underline{G}, \bar{G}]$. The support can be either bounded or unbounded.

Restricting $G$ to be strictly increasing ensures that $G$ is invertible, which is a key condition for identification of the model (Hotz and Miller 1993). The absolute continuity property of $G$ ensures that the dynamic programming model has a unique optimal solution almost surely.

\subsection{Parameters of interest}

Let $B$ be the set of all measurable, real-valued and bounded functions under sup norm. Then $B$ is clearly a Banach space. We assume that for $k \in\{0,1\}, u_{k}^{*}(x) \in B$. Then using Blackwell sufficient conditions (see Theorem 3.3 in Stockey and Lucas 1989), it can be shown that the value functions $v_{i}^{*}(x)$ are also in $B$ and are the unique fixed point of the system of Bellman equations (14) and (15). The parameters of interest in this model are therefore $\left(u_{k}^{*}(x), v_{k}^{*}(x), \beta, G, f_{x}\left(x^{\prime} \mid x, k\right)\right)$ for $k \in\{0,1\}$. We will refer to $v_{k}^{*}\left(x_{t}\right)$ as the derived structural parameters as they are derived from the primitive structural parameters $\left(u_{k}^{*}(x), \beta, G, f_{x}\left(x^{\prime} \mid x, k\right)\right)$ via the Bellman equations (14) and (15). Obtaining these structural parameters will then allow researchers to answer a variety of policy questions ${ }^{11}$. For example, the researcher may study a counterfactual policy experiment $\tau$ such that under policy $\tau$, the agent's behavior is generated from the new structure characterized by $\left(u_{k}^{* \tau}(x), v_{k}^{* \tau}(x), \beta^{\tau}, G^{\tau}, f_{x}^{\tau}\left(x^{\prime} \mid x, k\right)\right)^{12}$. Assuming the counterfactual structural parameters have a known mapping to the set of $\left(u_{k}^{*}(x), \beta, G, f_{x}\left(x^{\prime} \mid x, k\right)\right)$, then we can simulate the agent's behavior under the counterfactual policy if the set of structural parameters can be identified.

\footnotetext{
${ }^{10} \mathrm{As}$ in the static discrete choice models, the locations of the distributions for $\varepsilon_{1}$ and $\varepsilon_{0}$ are not separately identified since only the difference $\Delta \varepsilon$ matters in the conditional choice probability.

${ }^{11}$ Of course, we assume that the policy does not change the nature of the decision problem such that the agent still faces an optimal stopping time decision problem with the same absorbing choice.

${ }^{12}$ All variables superscripted with $\tau$ denote the same variables treated under the counterfactual policy $\tau$.
} 
Take a more concrete example from Aguirregabiria (2005). Consider the counterfactual policy is specified as $\tau_{k}(x)$, which may be some instantaneous tax benefit if the agent chooses alternative $k$. Then one interesting policy question would be to evaluate the conditional choice probability $P\left(\delta^{\tau}=1 \mid x\right)$ under this counterfactual policy. Another interesting aspect may be welfare analysis of such policy intervention and in this case, one could consider studying $E\left(v^{\tau}\left(s^{\prime}, \delta^{\tau}\right)-v\left(s^{\prime}, \delta\right) \mid x^{\prime}\right)^{13}$, which is the average impact of the policy. In this tax benefit example, $u_{k}^{* \tau}(x)=u_{k}^{*}(x)+\tau_{k}(x)$. In general $\tau_{k}(x)$ is known and specified in the policy experiment so that obtaining the deep structural parameters $u_{k}^{*}(x)$ allows us to identify the counterfactual parameter $u_{k}^{* \tau}(x)$. To simplify this example, we assume the policy does not change the remaining primitive parameters $\left(\beta, G, f_{x}\left(x^{\prime} \mid x, k\right)\right)^{14}$, then using equations (14) and (15), we can identify the counterfactual value function $v_{k}^{* \tau}(x)$ as follows.

$$
v_{1}^{* \tau}(x)=u_{1}^{* \tau}(x)+\beta E\left(v_{0}^{* \tau}\left(x^{\prime}\right) \mid x, d=1\right)+\beta\left[E\left(\Delta v^{* \tau}\left(x^{\prime}\right) \pi^{\tau}\left(s^{\prime}, 1\right) \mid x, d=1\right)+E\left(\Delta \varepsilon^{\prime} \pi^{\tau}\left(s^{\prime}, 1\right) \mid x, d=1\right)\right]
$$

and

$$
v_{0}^{* \tau}(x)=u_{0}^{* \tau}(x)+\beta E\left(v_{0}^{* \tau}\left(x^{\prime}\right) \mid x, d=0\right)
$$

Equations (16) and (17) illustrate a key difference in counterfactual policy experiment studies between static and dynamic models. In static models, $\tau_{k}(x)$ directly specifies the utility difference $u_{k}^{* \tau}(x)-u_{k}^{*}(x)$. However, in dynamic models $\tau_{k}(x) \neq v_{k}^{* \tau}(x)-v_{k}^{*}(x)$ since the agent is forward looking such that $\tau_{k}(x)$ will also affect the expected future values. Identification of the whole structure parameters will hence be sufficient to answer many interesting policy questions including those given in the example above ${ }^{15}$.

\section{Semiparametric identification of the optimal stopping time model}

We have a sample of individuals indexed by $i$ who follow the constituted structural optimal stopping time model. Data consist of the observed state variables and optimal choices for two consecutive periods $\left(\delta_{i}, x_{i}, \delta_{i}^{\prime}, x_{i}^{\prime}\right), i=$ $1,2, \ldots, n$, where $n$ denotes the sample size. Assume random sampling, we can suppress the individual index $i$. Under conditional independence (M2) assumption, the agent's belief $f_{x}\left(x^{\prime} \mid x, k\right)$ for $k \in\{0,1\}$ can be identified

\footnotetext{
${ }^{13}$ Indeed, $E\left(v\left(s^{\prime}, \delta\right) \mid x^{\prime}\right)$ is a dynamic version of McFadden's social surplus function. See also Rust $(1988,1994)$ for the properties of this surplus function.

${ }^{14}$ In other words, we assume $\left(\beta^{\tau}, G^{\tau}, f_{x}^{\tau}\left(x^{\prime} \mid x, k\right)\right)=\left(\beta, G, f_{x}\left(x^{\prime} \mid x, k\right)\right)$.

${ }^{15}$ If the counterfactual conditional choice probability is the main object of interest, then obtaining $u_{k}^{*}(x)$ is not necessary for identification. See Aguirregabiria $(2005,2006)$ for more discussions about identification of counterfactual conditional choice probability in the dynamic context.
} 
from the data $\left(\delta, x, x^{\prime}\right)$. To see this, with slight abuse of notation, let $x^{\prime}(k)$ be the value of $x^{\prime}$ when the control variable $d$ is evaluated at $k \in\{0,1\}$. In other words, $x^{\prime}(k)$ is the transition function that describes how next period state variable $x^{\prime}$ evolves given that the value of current control variable is $k$. M2 implies that the transition function $x^{\prime}(k)$ is not a function of $\varepsilon^{16}$. Therefore, $f_{x}\left(x^{\prime} \mid x, d=k\right)=f_{x}\left(x^{\prime}(k) \mid x\right)$. Note that $f_{x}\left(x^{\prime}(k) \mid x\right)$ is a counterfactual object since we observe $x^{\prime}(k)$ only for those individuals who actually chooses $k$. In other words, $x^{\prime}(k)$ is observed only when it is optimal to choose alternative $k$. We can identify $f_{x}\left(x^{\prime} \mid x, \delta=k\right)$ and

$$
f_{x}\left(x^{\prime} \mid x, \delta=k\right)=f_{x}\left(x^{\prime}(k) \mid x, \delta=k\right)=f_{x}\left(x^{\prime}(k) \mid x\right),
$$

where the second equality follows because the observed (optimal) choice $\delta$ evolves according to (4) and given $x, x^{\prime}(k)$ is independent of $\varepsilon$ under conditional independence assumption $\mathrm{M} 2^{17}$. Therefore, the agent's belief can be identified using the factual object $f_{x}\left(x^{\prime} \mid x, \delta=k\right)$ under M2, which is essentially the selection on observables assumption that is widely employed in the policy evaluation literature ${ }^{18}$ to identify the counterfactual objects of interest. Since $f_{x}\left(x^{\prime} \mid x, d=k\right)=f_{x}\left(x^{\prime} \mid x, \delta=k\right)$ is identified, we can replace the control variable (counterfactual) $d$ with the observed choice $\delta$ in Bellman equations (14) and (15). Using $\delta^{\prime}=\pi\left(s^{\prime}, \delta\right)$ and the assumption M2, we can further rewrite these two Bellman equations as

$$
v_{1}^{*}(x)=u_{1}^{*}(x)+\beta E\left(v_{0}^{*}\left(x^{\prime}\right) \mid x, \delta=1\right)+\beta\left[E\left(\Delta v^{*}\left(x^{\prime}\right) \delta^{\prime} \mid x, \delta=1\right)+E\left(\Delta \varepsilon^{\prime} \delta^{\prime} \mid x, \delta=1\right)\right]
$$

and

$$
v_{0}^{*}(x)=u_{0}^{*}(x)+\beta E\left(v_{0}^{*}\left(x^{\prime}\right) \mid x, \delta=0\right)
$$

Therefore, for the rest of the paper, we will direct work on the Bellman equations (19) and (20).

\subsection{Identification of the structural parameters when the distribution $G$ is known (Review of Magnac and Thesmar's identification analysis)}

Following Magnac and Thesmar (2002), we will first characterize the degree of identification for this optimal stopping time model.

\footnotetext{
${ }^{16}$ However, $x^{\prime}(k)$ may depend on current state variable $x$.

${ }^{17}$ Though $\delta_{t}$ depends on $\delta_{t-1}$ through its law of motion $(4), x_{t+1}(k)$ is independent of $\delta_{t-1}$ conditional on $x_{t}$ due to the assumption that the structural model.is first order Markov.

${ }^{18}$ See Heckman and Robb (1985) and Heckman, Lalonde, and Smith (1999) for surveys of policy evaluation literature based on counterfactual settings.
} 
Lemma 2 Under $M 4$, the conditional choice probability is $P\left(\delta^{\prime}=1 \mid x^{\prime}, \delta=1\right)=G\left(\Delta v^{*}\left(x^{\prime}\right)\right)$ so given $G, \Delta v^{*}$ can be identified. On the other hand, given $\Delta v^{*}, G$ is identified over the support of $\Delta v^{*}$.

Proof. Note that $P\left(\delta^{\prime}=1 \mid x^{\prime}, \delta=1\right)=P\left(\Delta v^{*}\left(x^{\prime}\right)+\Delta \varepsilon^{\prime} \geq 0 \mid x^{\prime}, \delta=1\right)$. Since $\Delta \varepsilon$ is serially independent and independent of the process of $x$, we have $P\left(\delta^{\prime}=1 \mid x^{\prime}, \delta=1\right)=G\left(\Delta v^{*}\left(x^{\prime}\right)\right)$. Under M4, $G$ is invertible so $\Delta v^{*}\left(x^{\prime}\right)=G^{-1}\left(P\left(\delta^{\prime}=1 \mid x^{\prime}, \delta=1\right)\right)$ is identified. On the other hand, if $\Delta v^{*}$ is given, then $G$ is identified as $P\left(\delta^{\prime}=1 \mid \Delta v^{*}\left(x^{\prime}\right), \delta=1\right)$ over the support of $\Delta v^{*}$.

Therefore, given $\beta$ and $u_{0}^{*}$, we can identify $v_{0}^{*}$ as the unique fixed point of the Bellman equation (20). Given $G$, we can identify $\Delta v^{*}$ using Lemma 2 and hence $v_{1}^{*}$ can also be identified. Then $u_{1}^{*}$ can be identified by plugging $v_{1}^{*}, v_{0}^{*}, \beta$ and $G$ into the Bellman equation (19).

Table 1 summarizes the identification analysis demonstrated above. Indeed this result is a special case of identification analysis of the general dynamic discrete choice model with known distribution of unobserved heterogeneity that has been studied in Magnac and Thesmar $(2002)^{19}$.

Table 1 : Degree of Identification of the dynamic optimal stopping time model

\begin{tabular}{|l|l|}
\hline Given & We can identify \\
\hline$G$ & $\Delta v^{*}$ \\
\hline$\beta$ and $u_{0}^{*}$ & $v_{0}^{*}$ \\
\hline$G, \beta$ and $u_{0}^{*}$ & $u_{1}^{*}, v_{1}^{*}, v_{0}^{*}$ \\
\hline
\end{tabular}

From Table 1 , we can see that $G$, the distribution of $-\Delta \varepsilon$, plays almost the most important role in this model not only because most of interesting structural parameters can be derived from $G$ but also because $G$ is indispensable when, besides identification of the structural parameters, the aim is to predict the conditional choice probability. Most empirical literature makes parametric assumptions on $G$. Though this facilitates the identification task in view of the results in Table 1, such parametric approach is rarely justified a priori and hence suffers from misspecification problems. Another remark on Table 1 is that obtaining $G$ alone is not sufficient to identify the complete structure of the model. We need the discounter factor $\beta$ and in particular the utility function for Choice $0^{20}$. Therefore, to identify the complete structure of the model, a natural alternative approach parallel to semiparametric methods of the static discrete choice models is to assume the systematic utilities while keeping the distribution of unobservables nonparametric. This paper aims to develop such semiparametric identification

\footnotetext{
${ }^{19}$ Note that this identification result still holds even all choices are recurrent (non-absorbing). See Magnac and Thesmar (2002) for proof of the general case.

${ }^{20}$ If time index is part of the state variables such that $u_{0}^{*}$ may change with time (for example, $u_{0}^{*}\left(t, x_{t}\right)=0$ for $t>T$ in finite horizon models), then the whole path of $u_{0}^{*}$ needs to be assumed. This can be regarded as the curse of dimensionality in the sense that the degree of under-identification gets more severe as the model horizon gets longer.
} 
results. In particular, we discuss the degree of identification in the case where only the path of utilities for the absorbing choice is assumed and then provide sufficient conditions that guarantee nonparametric identification of $G$, from which we can proceed to identify more structural parameters using the results in Table 1.

\subsection{Identification of the structural parameters when the distribution $G$ is unknown}

The Bellman equation (20) of the absorbing choice implies that once $\beta$ and $u_{0}^{*}$ are given, $v_{0}^{*}$ can be identified as the fixed point solution of this functional equation that does not depend on the distribution $G$. Therefore, by exploiting this behavioral restriction implied from the optimal stopping time model, it is natural to specify the instantaneous utility of the absorbing choice so that we can first obtain $v_{0}^{*}$ by solving equation (20). However, in contrast with the results in Table 1 , the following lemma shows that even assuming both $\beta$ and $u_{0}^{*}$, at best $v_{0}^{*}$ can be recovered and other structural parameters are still unidentified if there is no information about the distribution G.

Lemma 3 Assume $M_{4}$, given only $\beta$ and $u_{0}^{*}$, then $G, u_{1}^{*}$ and $v_{1}^{*}$ are not identified.

Proof. We assume $v_{0}^{*}$ is known since it can be identified in the model once $\beta$ and $u_{0}^{*}$ are given. Let $G$, $u_{1}^{*}$ and $v_{1}^{*}$ be a set of possible structure parameters that satisfy Bellman equation (19) and

$$
P\left(\delta^{\prime}=1 \mid x^{\prime}, \delta=1\right)=G\left(v_{1}^{*}\left(x^{\prime}\right)-v_{0}^{*}\left(x^{\prime}\right)\right)
$$

Consider another distribution $\widetilde{G} \neq G$. For example, one can take $\widetilde{G}(y)=[G(y)]^{\alpha}$ for some $\alpha>1$. Then the value function $\widetilde{v_{1}^{*}}\left(x^{\prime}\right) \equiv v_{0}^{*}\left(x^{\prime}\right)+\widetilde{G}^{-1}\left(G\left(v_{1}^{*}\left(x^{\prime}\right)-v_{0}^{*}\left(x^{\prime}\right)\right)\right)$ and $\widetilde{G}$ generate the same conditional choice probability as that generated by $v_{1}^{*}$ and $G$. Using Bellman equation (19), $\widetilde{v_{1}^{*}}\left(x^{\prime}\right)$ and $\widetilde{G}$ then implicitly define a utility function $\widetilde{u_{1}^{*}}$. Therefore, the parameters $\left(u_{1}^{*}, v_{1}^{*}, G\right)$ and $\left(\widetilde{u_{1}^{*}}, \widetilde{v_{1}^{*}}, \widetilde{G}\right)$ are observationally equivalent and they are not identified.

Lemma 3 says that assuming one value function alone provides no identification power. This result arises from lack of variation to distinguish between the unknown distribution $G$ and the unknown index function $v_{1}^{*}-v_{0}^{*}$. Therefore, $v_{1}^{*}$ and $G$ can be separately identified if we can fix $v_{1}^{*}$ but at the same time freely move $v_{0}^{*}$ which is an identifiable object when $\beta$ and $u_{0}^{*}$ are given so that we can trace the distribution $G$. As shown in Lemma 3 , there is no such variation-free condition for identification when $v_{1}^{*}$ and $v_{0}^{*}$ share completely the same set of regressors. Therefore, to achieve semiparametric identification when $G$ is nonparametric, it is useful to impose an exclusion restriction to provide such source of variation. In other words, if there is one continuous variable that is in the 
arguments of $v_{0}^{*}$ but is excluded from those of $v_{1}^{*}$, then we can separately identify $v_{1}^{*}$ and $G$ up to a location normalization. Theorem 4 demonstrates this semiparametric identification strategy.

Theorem 4 Let $x=(w, z)$ in which the subvectors $w$ and $z$ have no common component and both of them are non-empty. Denote the support of $x$ as $\Gamma_{X}=\Gamma_{W} \times \Gamma_{Z}$, where $\Gamma_{W}$ and $\Gamma_{Z}$ are the supports of $w$ and $z$, respectively. Let $u_{0}^{*}(x)=u_{0}^{*}(w, z) \in B$ be a known function. Assume also the following: (i) (excluded regressors) $u_{1}^{*}(x)=u_{1}^{*}(w)$ (ii) (conditional independence between observables) $\left(w^{\prime}, z^{\prime}\right) \perp z \mid w, d=1$ (iii) (continuous regressors) $\exists$ at least one continuous variable $z_{s}$ in the subvector $z$ such that the distribution of $z_{s}$ conditional on $w$ is non-degenerate and $v_{0}(x)$ is differentiable with respect to $z_{s}$ with $\frac{\partial v_{0}^{*}(x)}{\partial z_{s}} \neq 0$ almost surely over $\Gamma_{X}$. Then under $M 4$, given $\beta$, $v_{0}^{*}(x)=v_{0}^{*}(w, z)$ is identified and $v_{1}^{*}(x)=v_{1}^{*}(w)$ is identified up to a location normalization. The distribution $G$ is also identified up to a location normalization within the support of $\Delta v^{*}$.

Proof. Given $\beta$ and $u_{0}^{*}(x)=u_{0}^{*}(w, z) \in B$, we can identify $v_{0}^{*}(x)=v_{0}^{*}(w, z)$ by solving the fixed point of Bellman equation (20). Under assumptions (i) and (ii), the Bellman equation (19) implies that $v_{1}^{*}(x)=v_{1}^{*}(w)$. Consider any two pairs of the rest structural parameters $\left(u_{1}^{*}, v_{1}^{*}, G\right)$ and $\left(\widetilde{u_{1}^{*}}, \widetilde{v_{1}^{*}}, \widetilde{G}\right)$. If they are observationally equivalent, then following the proof in Lemma 3 we have

$$
\widetilde{v_{1}^{*}}\left(w^{\prime}\right) \equiv v_{0}^{*}\left(w^{\prime}, z^{\prime}\right)+\widetilde{G}^{-1}\left(G\left(v_{1}^{*}\left(w^{\prime}\right)-v_{0}^{*}\left(w^{\prime}, z^{\prime}\right)\right)\right)
$$

Equation (21) should hold for all values of $\left(w^{\prime}, z^{\prime}\right)$ in $\Gamma_{W} \times \Gamma_{Z}$. Let $\Lambda(y) \equiv \widetilde{G}^{-1}(G(y))$. M4 implies that $\Lambda$ is differentiable. So Under assumption (iii), we can take partial derivative of both sides in equation (21) with respect to $z_{s}$. Therefore, we have

$$
\frac{\partial v_{0}^{*}\left(w^{\prime}, z^{\prime}\right)}{\partial z_{s}}=\Lambda^{\prime}\left(v_{1}^{*}\left(w^{\prime}\right)-v_{0}^{*}\left(w^{\prime}, z^{\prime}\right)\right) \frac{\partial v_{0}^{*}\left(w^{\prime}, z^{\prime}\right)}{\partial z_{s}}
$$

Since $\frac{\partial v_{0}^{*}(x)}{\partial z_{s}} \neq 0$ almost surely, this implies $\Lambda^{\prime}(y)=1$ and hence $G(y)=\widetilde{G}(c+y)$ for all $y$ in the support of $\Delta v^{*}$ where the constant $c$ is a location parameter. Therefore, $G$ and $v_{1}^{*}$ are identified up to a location.

Setting the location parameter of $G$, for example the median of $G$, can normalize the location in Theorem 4. A direct implication of Theorem 4 is the identification of the partial derivatives of the value functions. These marginal values do not depend on the location normalization of value functions and may already be the structural objects of interest. For example, in doing welfare analysis, we may need $\partial E\left(v\left(x^{\prime}, \varepsilon^{\prime}\right) \mid x^{\prime}, \delta=1\right) / \partial x_{k}$, a measure to assess the impact of changing a particular state variable $x_{k}$ on the average social surplus function for the $\delta=1$ 
subpopulation. Note that

$$
\frac{\partial E\left(v\left(x^{\prime}, \varepsilon^{\prime}\right) \mid x^{\prime}, \delta=1\right)}{\partial x_{k}}=\frac{\partial v_{1}^{*}\left(x^{\prime}\right)}{\partial x_{k}} P\left(\delta^{\prime}=1 \mid x^{\prime}, \delta=1\right)+\frac{\partial v_{0}^{*}\left(x^{\prime}\right)}{\partial x_{k}} P\left(\delta^{\prime}=0 \mid x^{\prime}, \delta=1\right)^{21}
$$

is identified since the conditional choice probability is identified from the data and all the partial derivatives are identified under Theorem 4. In fact, using the control function approach, we can obtain direct identification of these derivatives. To see this, assume $v_{1}^{*}(w)$ and $v_{0}^{*}(w, z)$ are differentiable with respect to some continuous component $w_{r}$. The conditional choice probability equation is

$$
P\left(\delta^{\prime}=1 \mid w^{\prime}, z^{\prime}, \delta=1\right)=G\left(v_{1}^{*}\left(w^{\prime}\right)-v_{0}^{*}\left(w^{\prime}, z^{\prime}\right)\right)=P\left(\delta^{\prime}=1 \mid w^{\prime}, v_{0}^{*}\left(w^{\prime}, z^{\prime}\right), \delta=1\right)
$$

Take partial derivative with respect to $w_{r}$ and get

$$
\frac{\partial P\left(\delta^{\prime}=1 \mid w^{\prime}, z^{\prime}, \delta=1\right)}{\partial w_{r}}=g\left(v_{1}^{*}\left(w^{\prime}\right)-v_{0}^{*}\left(w^{\prime}, z^{\prime}\right)\right)\left[\frac{\partial v_{1}^{*}\left(w^{\prime}\right)}{\partial w_{r}}-\frac{\partial v_{0}^{*}\left(w^{\prime}, z^{\prime}\right)}{\partial w_{r}}\right] 22
$$

On the other hand, we can identify $g\left(v_{1}^{*}\left(w^{\prime}\right)-v_{0}^{*}\left(w^{\prime}, z^{\prime}\right)\right)$ by taking derivative with respect to $v_{0}^{*}$ as follows.

$$
\frac{\partial P\left(\delta^{\prime}=1 \mid w^{\prime}, v_{0}^{*}\left(w^{\prime}, z^{\prime}\right), \delta=1\right)}{\partial v_{0}^{*}}=-g\left(v_{1}^{*}\left(w^{\prime}\right)-v_{0}^{*}\left(w^{\prime}, z^{\prime}\right)\right)
$$

So putting (25) and (26) together, we have

$$
\frac{\partial v_{1}^{*}\left(w^{\prime}\right)}{\partial w_{r}}=-\left(\frac{\partial P\left(\delta^{\prime}=1 \mid w^{\prime}, z^{\prime}, \delta=1\right)}{\partial w_{r}}\right) /\left(\frac{\partial P\left(\delta^{\prime}=1 \mid w^{\prime}, v_{0}^{*}\left(w^{\prime}, z^{\prime}\right), \delta=1\right)}{\partial v_{0}^{*}}\right)+\frac{\partial v_{0}^{*}\left(w^{\prime}, z^{\prime}\right)}{\partial w_{r}}
$$

Therefore, identification of $\frac{\partial v_{1}^{*}\left(w^{\prime}\right)}{\partial w_{r}}$ immediately follows since given $\beta$ and $u_{0}^{*}$ the right hand side objects of $(27)$ are identified.

The assumptions of excluded regressors (i) and conditional independence (ii) in Theorem 4 can produce the required exclusion restriction between the two value functions so that we can distinguish the source of variation between the unknown $v_{1}^{*}$ and $G$ by moving only the excluded variables. Assumption (i) may be justified if there are choice specific attributes. Note that assumptions (i) does not preclude common attributes since the vector of attributes $w$ is allowed to appear in $u_{0}^{*}$ and $v_{0}^{*}$. Only the attributes $z$ are excluded. However, assumption (i)

\footnotetext{
${ }^{21}$ Since the model implies that $E\left(v\left(x^{\prime}, \varepsilon^{\prime}\right) \mid x^{\prime}, \delta=1\right)=\int \max \left(v_{1}^{*}\left(x^{\prime}\right)+\varepsilon_{1}^{\prime}, v_{0}^{*}\left(x^{\prime}\right)+\varepsilon_{0}^{\prime}\right) d F\left(\varepsilon^{\prime}\right)$, this formula follows by interchanging the integral and differentiation, which can be justified under Lebesgue Dominated Convergence Theorem when both $\frac{\partial v_{1}^{*}(x)}{\partial x_{k}}$ and $\frac{\partial v_{0}^{*}(x)}{\partial x_{k}}$
are bounded.

${ }^{22}$ Recall that $g$ is the density of $-\Delta \varepsilon$
} 
alone is not sufficient to generate the required exclusion restriction in the conditional choice probability equation since the choice specific attributes may enter both value functions via the information set that the agent uses to form their expected future value. Assumption (ii) is sufficient to remove such effect by regulating the predicability of these attributes through conditional independence assumption. A sufficient condition to validate assumption (ii) is the case in which the transition probability density $f\left(w^{\prime}, z^{\prime} \mid w, z, \delta=1\right)$ can be factored out as the product of $f\left(z^{\prime} \mid w^{\prime}, \delta=1\right) f\left(w^{\prime} \mid w, \delta=1\right)$. Hence assumption (ii) essentially requires that once conditional on $\delta=1, w$ serves as a sufficient statistic for predicting all observed state variables of next period. Note that semiparametric identification in Theorem 4 can be achieved by requiring only one excluded variable $z$ and over-identification may arise if more than one exclusion restrictions are available. Therefore, the identification restrictions stated in Theorem 4 may not be very demanding for practical applications.

Theorem 4 requires $u_{0}^{*}$ is specified a priori. However, complete specification of the instantaneous utility function $u_{0}^{*}$ may not be necessary in the sense that one can specify it up to finite dimensional unknown parameters. Of course, if $u_{0}^{*}$ depends on some unknown parameters, one also needs to guarantee these parameters are identified. Let $u_{0}^{*}(x)=u_{0}^{*}(w, z ; \theta)$ be known up to a finite $J$-dimensional vector of parameters, $\theta \in \Theta$, where $\Theta$ is a compact subset of $R^{J}$. It is not easy to characterize the value function $v_{0}^{*}(x ; \theta)$ a priori even though the shape of $u_{0}^{*}(x)$ is given. This is due to the nonparametric transition probability density $f\left(x^{\prime} \mid x, \delta=0\right)$ that operates in the Bellman equation (20) and hence also determines the shape of $v_{0}^{*}(x ; \theta)$. Instead of providing identification of $\theta$ in the general case, we will give the identification result for some popular specifications of utility functions ${ }^{23}$.

Theorem 5 Assume all conditions in Theorem \& still hold except that $u_{0}^{*}(x)=u_{0}^{*}(w, z ; \theta)=\theta^{\prime} h(w, z)$ where $h(w, z)$ is a J-dimensional vector of known functions $h_{j}(w, z) \in B$ and each component $\theta_{j}$ in the vector $\theta$ is not zero. Then $v_{0}^{*}(x)=\theta^{\prime} r(w, z)$, where $r(w, z)$ is a J-dimensional vector of functions $r_{j}(w, z)$ with each $r_{j}(w, z) \in B$ satisfying the Bellman equation $r_{j}(w, z)=h_{j}(w, z)+\beta E\left(r_{j}\left(w^{\prime}, z^{\prime}\right) \mid w, z, \delta=0\right)$. Hence, given $\beta, \theta$ is identified up to a scale normalization.

Proof. Given $u_{0}^{*}(x)=u_{0}^{*}(w, z ; \theta)=\theta^{\prime} h(w, z)$, we shall first verify the conjecture that $v_{0}^{*}(x)=\theta^{\prime} r(w, z)$ does satisfy Bellman equation (20). Plugging $v_{0}^{*}(x)=\theta^{\prime} r(w, z)$ into equation (20), we have

$$
\theta^{\prime} r(x)=\theta^{\prime} h(x)+\beta E\left(\theta^{\prime} r\left(x^{\prime}\right) \mid x, \delta=0\right)=\theta^{\prime}\left(h(x)+\beta E\left(r\left(x^{\prime}\right) \mid x, \delta=0\right)\right)
$$

\footnotetext{
${ }^{23}$ We leave the identification analysis of a general specification $u_{0}^{*}(w, z ; \theta)$ for further research.
} 
Therefore,

$$
r(x)=h(x)+\beta E\left(r\left(x^{\prime}\right) \mid x, \delta=0\right)
$$

Since $h(x) \in B, r(x)$ is then the unique fixed point of Bellman equation (29). So $v_{0}^{*}(x)=\theta^{\prime} r(x)$ is the unique fixed point of Bellman equation (20). Since $h(x)$ is known, given $\beta, r(x)$ is then identified using equation (29) and hence $v_{0}^{*}(x)$ is identified up to $\theta$. So the conditional choice probability in this case is

$$
\begin{aligned}
P\left(\delta^{\prime}\right. & \left.=1 \mid w^{\prime}, z^{\prime}, \delta=1\right)=G\left(v_{1}^{*}\left(w^{\prime}\right)-v_{0}^{*}\left(w^{\prime}, z^{\prime}\right)\right) \\
& =G\left(v_{1}^{*}\left(w^{\prime}\right)-\theta^{\prime} r\left(w^{\prime}, z^{\prime}\right)\right)=P\left(\delta^{\prime}=1 \mid w^{\prime}, r\left(w^{\prime}, z^{\prime}\right), \delta=1\right)
\end{aligned}
$$

Taking partial derivative with respect to $r_{j}\left(w^{\prime}, z^{\prime}\right)$, we have

$$
\frac{\partial P\left(\delta^{\prime}=1 \mid w^{\prime}, r\left(w^{\prime}, z^{\prime}\right), \delta=1\right)}{\partial r_{j}}=-g\left(v_{1}^{*}\left(w^{\prime}\right)-\theta^{\prime} r\left(w^{\prime}, z^{\prime}\right)\right) \theta_{j}
$$

Using the average derivative arguments, we have

$$
E\left(\frac{\partial P\left(\delta^{\prime}=1 \mid w^{\prime}, r\left(w^{\prime}, z^{\prime}\right), \delta=1\right)}{\partial r_{j}}\right)=-E\left(g\left(v_{1}^{*}\left(w^{\prime}\right)-\theta^{\prime} r\left(w^{\prime}, z^{\prime}\right)\right)\right) \theta_{j}
$$

Therefore, $\theta_{j}$ is identified up to a scale normalization.

Theorem 5 provides the identification result when the instantaneous utility $u_{0}^{*}$ is specified as linear in parameters. Note that in order to provide the required exclusion restriction, each $r_{j}(w, z)$ in Theorem 5 needs to be a non-trivial function in $z$. This requires either each $h_{j}(w, z)$ is a non-trivial function in $z$ or the transition probability $p\left(w^{\prime}, z^{\prime} \mid w, z, \delta=0\right)$ varies with $z$. Therefore, together with the exclusion restriction imposed in Theorem 4 , it is more clear to note that the variable $z$ essentially serves as the "instrumental variable" in semiparametric identification of this structural model in the sense that it provides variation for the identifiable endogenous object $v_{0}^{*}(w, z)$ (the "rank condition") but cannot affect the "unobserved" object $v_{0}^{*}(w)$ (the "exclusion" condition). Theorem 5 adopts the usual average derivative approach to identify the unknown parameters $\theta$. As in static discrete choice models, the average derivative method can at best identify the unknown parameters up to scale normalization. Such scale normalization is innocuous in discrete choice models since expected utility theory implies that one can only identify an agent's preferences up to a positive linear transformation (Rust 1994). Once the scale of $\theta$ is determined, using Theorem 4 we can proceed to identify $v_{0}^{*}, v_{1}^{*}$ and $G$ over the support of $\Delta v^{*}$. 
To identify the remaining structural parameter $u_{1}^{*}(x)$, note that from the Bellman equation (19), we have

$$
u_{1}^{*}(x)=v_{1}^{*}(x)-\beta E\left(v_{0}^{*}\left(x^{\prime}\right) \mid x, \delta=1\right)-\beta\left[E\left(\Delta v^{*}\left(x^{\prime}\right) \delta^{\prime} \mid x, \delta=1\right)+E\left(\Delta \varepsilon^{\prime} \delta^{\prime} \mid x, \delta=1\right)\right]
$$

Given $\beta$, after applying previous theorems, all righthand side objects except the last term of equation (33) can be identified. The term $E\left(\Delta \varepsilon^{\prime} \delta^{\prime} \mid x, \delta=1\right)$ may not be identified because the value functions are assumed bounded in this model so that the support of the distribution $G$ may not be completely contained in the support of $\Delta v^{*}$. In other words, $u_{1}^{*}(x)$ is not identified unless the support of $G$ is a subset of the support of $\Delta v^{*}$ or more information about the tail behavior (outside the support of $\Delta v^{*}$ ) of $G$ is available. By further investigating the unidentified term $E\left(\Delta \varepsilon^{\prime} \delta^{\prime} \mid x, \delta=1\right)$, although not point identified, we can show that the upper bound of $u_{1}^{*}(x)$ can be identified. To see this, let the support of $\Delta v^{*}$ be $\left[L_{v}, U_{v}\right]$ and note that

$$
\begin{aligned}
E\left(\Delta \varepsilon^{\prime} \delta^{\prime} \mid x, \delta\right. & =1)=E\left(\Delta \varepsilon^{\prime} \mid \delta^{\prime}=1, x, \delta=1\right) P\left(\delta^{\prime}=1 \mid x, \delta=1\right) \\
& =E\left(E\left(\Delta \varepsilon^{\prime} \mid \delta^{\prime}=1, x^{\prime}, x, \delta=1\right) \mid \delta^{\prime}=1, x, \delta=1\right) P\left(\delta^{\prime}=1 \mid x, \delta=1\right) \\
& =E\left(E\left(\Delta \varepsilon^{\prime} \mid \Delta v^{*}\left(x^{\prime}\right)+\Delta \varepsilon^{\prime} \geq 0, x^{\prime}, x, \delta=1\right) \mid \delta^{\prime}=1, x, \delta=1\right) P\left(\delta^{\prime}=1 \mid x, \delta=1\right) \\
& =-\left[\int_{\Gamma_{x}} \frac{\int_{-\infty}^{\Delta v^{*}(\tau)}\left(-\Delta \varepsilon^{\prime}\right) g\left(-\Delta \varepsilon^{\prime}\right) d\left(-\Delta \varepsilon^{\prime}\right)}{P\left(\delta^{\prime}=1 \mid x^{\prime}=\tau, \delta=1\right)} f\left(\tau \mid \delta^{\prime}=1, x, \delta=1\right) d \tau\right] P\left(\delta^{\prime}=1 \mid x, \delta=1\right)
\end{aligned}
$$

We can further analyze the term $\int_{-\infty}^{\Delta v^{*}(\tau)}\left(-\Delta \varepsilon^{\prime}\right) g\left(-\Delta \varepsilon^{\prime}\right) d\left(-\Delta \varepsilon^{\prime}\right)$ in (34) as follows. Let $\eta=-\Delta \varepsilon^{\prime}$ and $W \equiv$ $\int_{-\infty}^{L_{v}} G(\eta) d \eta$. Then

$$
\begin{aligned}
\int_{-\infty}^{\Delta v^{*}(\tau)}\left(-\Delta \varepsilon^{\prime}\right) g\left(-\Delta \varepsilon^{\prime}\right) d\left(-\Delta \varepsilon^{\prime}\right) & =\int_{-\infty}^{\Delta v^{*}(\tau)} \eta g(\eta) d \eta=\int_{-\infty}^{L_{v}} \eta g(\eta) d \eta+\int_{L_{v}}^{\Delta v^{*}(\tau)} \eta g(\eta) d \eta \\
& =L_{v} G\left(L_{v}\right)+\int_{L_{v}}^{\Delta v^{*}(\tau)} \eta g(\eta) d \eta-W
\end{aligned}
$$

Note that the value of $W$ is not identified since the distribution $G$ is not identified for $\eta \notin\left[L_{v}, U_{v}\right]$. However, the "bias" term $W$ is non-negative and shrinks to zero when the lower bound of the support of $\Delta v^{*}$ approach $\underline{G}$, the lower bound of the support of $G$. Plugging (35) into (34), we have

$$
\left.E\left(\Delta \varepsilon^{\prime} \delta^{\prime} \mid x, \delta=1\right)=\left(W-L_{v} G\left(L_{v}\right)\right) E\left(\frac{\delta^{\prime}}{P\left(\delta^{\prime}=1 \mid x^{\prime}, \delta=1\right)} \mid x, \delta=1\right)\right)-A P\left(\delta^{\prime}=1 \mid x, \delta=1\right),
$$


where $A$ is defined as

$$
A=\int_{\Gamma_{x}} \frac{\int_{L_{v}}^{\Delta v^{*}(\tau)} \eta g(\eta) d \eta}{P\left(\delta^{\prime}=1 \mid x^{\prime}=\tau, \delta=1\right)} f\left(\tau \mid \delta^{\prime}=1, x, \delta=1\right) d \tau
$$

Since $W$ is non-negative, setting $W=0$ will give a lower bound of $E\left(\Delta \varepsilon^{\prime} \delta^{\prime} \mid x, \delta=1\right)$ and hence the upper bound of $u_{1}^{*}(x)$ is identified. Therefore, treating the tail area of $G$ as zero (setting $W=0$ ) gives a nonparametric approximation of $u_{1}^{*}(x)$ and this approximation gets more precise when $G$ is less heavy-tailed. In practice, one would expect that the bias $W$ does not matter if the support of $\Delta v^{*}$ is wide enough.

It is clear that given the discount factor $\beta, W$ can be identified if one is willing to parameterize $u_{1}^{*}(x)$. For example, if $u_{1}^{*}(x)=u_{1}^{*}(x, \alpha)$ for some finite dimensional vector of parameters $\alpha$, then $W$ and $\alpha$ can be (over-) identified by plugging (36) into (33) and then solving for $W$ and $\alpha^{24}$. Table 2 summarizes the semiparametric identification results developed in this paper.

Table 2 : Semiparametric Identification of the dynamic optimal stopping time model

\begin{tabular}{|l|l|}
\hline Key conditons & We can identify \\
\hline$\beta, u_{0}^{*}(x)$ & $v_{0}^{*}(x)$ \\
\hline$\beta, u_{0}^{*}(x)$, and excluded variables & $v_{0}^{*}(x), v_{1}^{*}(x), G$ over $\left[L_{v}, U_{v}\right]$, and upper bound of $u_{1}^{*}(x)$ \\
\hline
\end{tabular}

\subsection{Illustrating examples : disposal of capital and retirement from labor force}

We present in this section two motivating examples in which the analysis of this paper may be useful.

\subsubsection{A model of capital disposal}

Consider the following microeconometric capital retirement model ${ }^{25}$. A firm produces a good using a machine. Assume the industry is competitive so that the unit price of the good $p_{t}$ at every period is exogenous to the firm's decision. At every period, the firm decides whether or not to retire the machine. Assuming the machine is on at its full capacity $k_{t}$ when the firm decides to keep and operate the machine. Operating at full capacity may be due to the high cost of adjusting the machine's utilization level. Alternatively, we can regard $k_{t}$ as the optimized level of utilization when the machine is operated so that $p_{t} k_{t}$ is interpreted as the per period indirect profit function. When the machine is on, there is also associated maintenance or operating cost $c\left(k_{t}, a_{t}, w_{t}, l_{t}\right)$, where $a_{t}$ is the age of the machine, $w_{t}$ is the wage of the engineer who looks after the machine, and $l_{t}$ is the number of engineers required to operate the machine. Let Choice 1 be the decision of keeping and operating the machine and Choice

\footnotetext{
${ }^{24}$ Of course, the usual rank condition should be satisfied to guarantee a unique solution of $W$ and $\alpha$.

${ }^{25}$ See Das (1992) for another similar but more detailed structural capital retirement model.
} 
0 be retiring and scrapping it. Then we have $u_{1}\left(x, \varepsilon_{1}\right)=p k-c(k, a, w, l)+\varepsilon_{1}$ and hence $u_{1}^{*}(x)=p k-c(k, a, w, l)$. When the machine is retired, the firm receives its scrapping value. Decompose the scrapping value of the machine as $z_{t}+\varepsilon_{2, t}$, where $z_{t}$ is the booked (estimated) remaining value of the machine that may be observed on the firm's accounting balance sheet and $\varepsilon_{2, t}$ is the unobservable that accounts for unobserved disposal cost or any discrepancy betweeb $z_{t}$ and the actual selling value of the machine. Then in our terminology we have $u_{0}\left(x, \varepsilon_{2}\right)=z+\varepsilon_{2}$ and $u_{0}^{*}(x)=z$. The parameters of interest are the nonparametric cost function $c(k, a, w, l)$ and the value functions $v_{0}^{*}(x)$ and $v_{1}^{*}(x)$. To apply the developed identification strategy, we need to assume the excluded variable $z_{t}$ satisfies the key conditional independence assumption stated in Theorem $4^{26}$. This assumption may be satisfied when the firm estimates the current remaining value of the machine based on its generated revenue and operation cost in the past plus some independent random noise that reflects the firm's reassessment to the current status of the machine. When conditions in Theorem 4 are fulfilled, we can identify the cost function if the support of the distribution $G$ is in the support of $\Delta v^{*}$ or the lower bound of the cost function if $G$ has unbounded support.

\subsubsection{A model of retirement behavior}

Assuming a retirement model in which at every period the agent needs to decide whether to continue working $\left(d_{t}=1\right)$ or to retire from the labor force $\left(d_{t}=0\right)$. Once the agent retires, he stay retired forever. Therefore, the retirement decision is absorbing. The instantaneous utility of working is $u_{1}^{*}(w)$, where $w$ denotes the wage. On the other hand, the instantaneous utility of retiring is $u_{0}^{*}(p)$, where $p$ denotes the pension ${ }^{27}$. The agent is forward-looking and he will form expectation of future level of wage and pension conditional on current state variables that are relevant for prediction. The rest of relevant state variables are his current position or rank in this system denoted as $r$ and his current tenure denoted as $l$. The transition equations of the observed state variables in this system are assumed as follows.

$$
\begin{aligned}
& \text { Wage : } w^{\prime}=d\left(h_{w}\left(w, l, r, \eta_{w}^{\prime}\right)\right) \\
& \text { Tenure }: l^{\prime}=l+d \\
& \text { rank : } r^{\prime}=d\left(h_{r}\left(r, l, \eta_{r}^{\prime}\right)\right)+(1-d) r \\
& \text { pension }: p^{\prime}=h_{p}\left(l, r, \eta_{p}^{\prime}\right),
\end{aligned}
$$

where $h_{w}, h_{r}$, and $h_{p}$ are deterministic functions that represent the wage, rank, and pension schedules. $\eta_{w}$, $\eta_{r}$, and $\eta_{p}$ are mutually and serially independent random noises that reflect the unexpected deviations from the

\footnotetext{
${ }^{26}$ Indeed, the conditional independence assumption in Theorem 4 is testable because this restriction is imposed on observed state variables.

${ }^{27}$ The pension variable is always observed since in most cases, even if the agent stays at work, he can obtain information about his scheduled pension at each period from his pension administrators.
} 
specified schedules of the wage, rank, and pension variables, respectively. Note that this simple setup can catch the key features of a tenure-based working system in which workers' wage and promotion of rank are mainly based on workers' tenure. Typical examples for this kind of system are public servants, military personnel, and teachers who are paid by the government. Then under such a system, $\left(w^{\prime}, l^{\prime}, r^{\prime}, p^{\prime}\right) \perp p \mid w, l, r, \delta=1^{28}$ so that we can adopt Theorem 4 to identify this model. Note that the key source of identification in this example comes from the sticky design of the pension schedule. If the agent is allowed to invest his pension in some financial assets, then his pension will depend on the performance of the financial market so that $p$ will be serially correlated, which then invalidates the conditional independence assumption. However, as the developed identification strategy requires only one excluded variable that can validate the conditional independence condition, if some part of the pension schedule is sticky, then the availability of the information of this part can still provide identification. Intuitively speaking, as the instrumental variable approach, the identification power comes from the sticky institional feature that affects value function of choosing to retire but does not affect that of choosing to work.

\section{Conclusions}

This paper develops semiparametric identification results for a structural dynamic optimal stopping time model. The main parametric assumption for this semiparametric identification method is the parametric specification of the per-period return function associated with the absorbing choice. The distribution of unobserved state variables and per-period return function for non-absorbing choice are both nonparametric. The identification crucially depends on the availability of at least one excluded continuous variable that affect the value function of the absorbing choice but not that of the non-absorbing choice. The implementation of the identification method developed in this paper will result in mutli-stage estimation procedure. In the first stage, one needs to obtain the value function for the absorbing choice. This requires solving a contraction mapping for the empirical counterpart of Bellman equation (20). Note that there is no unobserved component in equation (20) so one can save computational complexity arising from integrating out the unobservables as in the usual discrete choice models. In the second stage, one can use the estimated value function of the absorbing choice as the control function to estimate the value function of the non-absorbing choice.

\section{Reference}

\footnotetext{
${ }^{28}$ Recall that we can replace the counterfactual control variable $d$ with the observed choice $\delta$ under the conditional independence assumption M2.
} 
1. Adda, J. and Cooper R. (2000), "Balladurette and Juppette: A Discrete Analysis of Scrapping Subsidies", Journal of Political Economy, 108, 778-806.

2. Aguirregabiria, V. (2005), "Nonparametric Identification of Behavioral Responses to Counterfactual Policy Interventions in Dynamic Discrete Decision Processes", Economics Letters, 87, 393-398.

3. Aguirregabiria, V. (2006), "Another Look at the Identification of Dynamic Discrete Decision Processes, with an Application to Retirement Behavior", unpublished manuscript, University of Toronto.

4. Berkovec, J. and Stern, S. (1991), "Job Exit Behavior of Old Man", Econometrica, 59, 189-210.

5. Cameron, S. and Heckman, J. (1998), "Life-Cycle Schooling and Dynamic Selection Bias: Models and Evidence for Five Cohorts of American Males", Journal of Political Economy, 106, 262-333.

6. Cooper, R., Haltiwanger, J. and Power, L. (1999), "Machine Replacement and the Business Cycle: Lumps and Bumps", American Economic Review, 89, 921-946.

7. Cosslett, S. R. (1983), "Distribution-free Maximum Likelihood Estimator of the Binary Choice Model", Econometrica, 51, 765-782.

8. Daula, T. and Moffitt, R. (1995), "Estimating Dynamic Models of Quit Behavior: The Case of Military Reenlistment", Journal of Labor Economics, 13, 499-523.

9. Das, S. (1992), "A Micro-Econometric Model of Capital Utilization and Retirement: the Case of the U.S. Cement Industry", Review of Economic Studies, 59, 277-297.

10. Eckstein, Z. and Wolpin, K. (1989), "The Specification and Estimation of Dynamic Stochastic Discrete Choice Models: A Survey", Journal of Human Resources, 24, 562-598.

11. Eckstein, Z. and Wolpin, K. (1999), "Why Youth Drop out of High School: The Impact of Preferences, Opportunities and Abilities", Econometrica, 67, 1295-1339.

12. Erdem, T. and Keane, M. (1996), "Decision-Making Under Uncertainty: Capturing Dynamic Brand Choice Processes in Turbulent Consumer Goods Markets", Marketing Science, 15, 1-20.

13. Heckman, J., LaLonde, R. and Smith, J. (1999), "The Economics and Econometrics of Active Labor Market Programs", In O. Ashenfelter, and D. Card, (eds), The Handbook of Labor Economics, New York: NorthHolland. 
14. Heckman, J. and Navarro, S. (2007), "Dynamic Discrete Choice and Dynamic Treatment Effects", Journal of Econometrics, 136,341-396.

15. Heckman, J. and Robb, R. (1985), "Alternative Methods for Evaluating the Impact of Interventions", In J. Heckman, and B. Singer, (eds), Longitudinal Analysis of Labor Market Data, Cambridge University Press.

16. Hotz, V. J. and Miller, R. (1993), "Conditional Choice Probabilities and the Estimation of Dynamic Models", Review of Economic Studies, 60, 497-529.

17. Ichimura, H. (1993), "Semiparametric least squares (SLS) and weighted SLS estimation of single-index models", Journal of Econometrics, 58, 71-120.

18. Karlstrom, A., Palm, M. and Svensson, I. (2004), "A Dynamic Programming Approach to Model the Retirement Behavior of Blue-Collar Workers in Sweden", Journal of Applied Econometrics, 19, 795-807.

19. Keane, M. and Wolpin, K. (1997), "The Career Decisions of Young Men", Journal of Political Economy, $105,473-521$.

20. Klein, R. and Spady, R. (1993), "An Efficient Semiparametric Estimator for Binary Response Models", Econometrica, 61, 387-421.

21. Lewbel, A. and Linton, O. (2005), "Nonparametric Matching and Efficient Estimators of Homothetically Separable Functions", manuscript.

22. Magnac, T. and Thesmar, D. (2002), "Identifying Dynamic Discrete Decision Processes", Econometrica, 70, 801-816.

23. Manski, C. F. (1975), "Maximum Score Estimation of the Stochastic Utility Model of Choice", Journal of Econometrics, 3, 205-228.

24. Manski, C. F. (1985), "Semiparametric Analysis of Discrete Responses: Asymptotic Properties of the Maximum Score Estimator", Journal of Econometrics, 27,313-334.

25. Manski, C. F. (1993), "Dynamic Choices in Social Settings", Journal of Econometrics, 58, 121-136.

26. Matzkin, R. (1991), "Semiparametric Estimation of Monotone and Concave Utility Functions for Polychotomous Choice Models", Econometrica, 59, 1315-1327. 
27. Matzkin, R. (1992), "Nonparametric and Distribution-Free Estimation of the Binary Threshold Crossing and the Binary Choice Models", Econometrica, 60, 239-270.

28. Miller, R. (1984), "Job Matching and Occupational Choice", Journal of Political Economy, 92, 1086-1120.

29. Pakes, A. (1986), "Patents as Options: Some Estimates of the Value of Holding European Patent Stocks", Econometrica, 54, 755-784.

30. Rothwell, G. and Rust, J. (1997), "On the Optimal Lifetime of Nuclear Power Plants", Journal of Business and Economic Statistics, 15, 195-208.

31. Rust, J. (1987), "Optimal Replacement of GMC Bus Engines: An Empirical Model of Harold Zurcher", Econometrica, 57, 1027-1058.

32. Rust (1988), "Maximum Likelihood Estimation of Discrete Control Processes", SIAM Journal of Control and Optimization, 26, 1006-1024.

33. Rust, J. (1994), "Structural Estimation of Markov Decision Processes", in Handbook of Econometrics, Vol. 4, ed. by R. Engle and D. McFadden, Amsterdam: North Holland, 3081-3143.

34. Rust, J. and Phelan, C. (1997), "How Social Security and Medicare Affect Retirement Behavior in a World of Incomplete Markets", Econometrica, 65, 781-832.

35. Stoker, T. M. (1986), "Consistent Estimation of Scaled Coefficients", Econometrica, 54, 1461-1481.

36. Stokey, N. and Lucas, R. (1989), "Recursive Methods in Economics Dynamics", Harvard University Press, Cambridge, Massachusetts.

37. Taber, C. (2000), "Semiparametric Identification and Heterogeneity in Discrete Choice Dynamic Programming Models", Journal of Econometrics, 96, 201-229.

38. Vytlacil, E. (2002), "Independence, Monotonicity, and Latent Index Models: An Equivalence Result", Econometrica, 70, 331-341.

39. Vytlacil, E. (2005), "A Note on Additive Separability and Latent Index Models of Binary Choice: Representation Results", unpublished manuscript, Standford University.

40. Wolpin, K. (1984), "An Estimable Dynamic Stochastic Model of Fertility and Child Mortality", Journal of Political Economy, 92, 852-874. 
41. Wolpin, K. (1987), "Estimating A Structural Search Model: The Transition From School To Work", Econometrica, 55, 801-817.

42. Wolpin, K. (1996), "Public Policy Uses of Discrete Choice Dynamic Programming Models", American Economic Review, 86, 427-432. 